\title{
Diacronie
}

Studi di Storia Contemporanea

$\mathrm{N}^{\circ} 26,2$ | 2016

Un bilancio della scommessa democratica della Chiesa cattolica

\section{De la Dignitatis Humanae a la democracia: la crisis de la Acción Católica y el nacimiento de PROLESA (1965-1978)}

\section{Carlos González Martínez}

\section{OpenEdition}

\section{Journals}

Edición electrónica

URL: http://journals.openedition.org/diacronie/4121

DOI: 10.4000/diacronie.4121

ISSN: 2038-0925

Editor

Association culturelle Diacronie

Referencia electrónica

Carlos González Martínez, « De la Dignitatis Humanae a la democracia: la crisis de la Acción Católica y el nacimiento de PROLESA (1965-1978) », Diacronie [En línea], № 26, 2 | 2016, documento 5, Puesto en línea el 29 junio 2016, consultado el 20 abril 2019. URL : http://journals.openedition.org/ diacronie/4121; DOI : 10.4000/diacronie.4121 


\section{Diacronie}

\section{De la Dignitatis Humanae a la democracia: la crisis de la Acción Católica y el nacimiento de PROLESA (1965-1978)}

\section{Carlos GONZÁLEZ MARTÍNEZ *}

El Concilio Vaticano II, y más en concreto la Dignitatis Humanae, marcó el inicio de la lenta separación de la Iglesia con el régimen del general Franco. En el ámbito seglar, ese proceso se llevó a cabo fundamentalmente en el episodio conocido como la "Crisis de la Acción Católica". Tras varios años caracterizados por la falta de entendimiento entre la Conferencia Episcopal y los dirigentes del apostolado, estos últimos dimitieron en 1968. A continuación, bajo la dirección de Salvador Sánchez-Terán fundaron PROLESA, una asociación que sirvió como cobertura legal para la formación de un grupo de apertura a la democracia. En este artículo pretendemos profundizar en el estudio de esos acontecimientos, así como mostrar el papel de esas personas en la Transición española.

\section{Introducción}

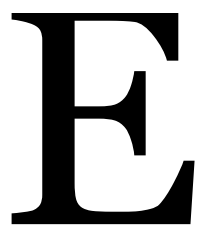

n mayo de 1968, como consecuencia de la falta de entendimiento entre la jerarquía de la Iglesia española y los dirigentes del apostolado seglar, se produjo la dimisión masiva de buena parte de estos últimos. El fenómeno conocido como "la crisis de la Acción Católica" puso en evidencia las discrepancias entre los defensores de la continuidad del nacionalcatolicismo, si bien algo adaptado a la nueva doctrina del Vaticano II, y los partidarios de aplicar, con todas sus consecuencias, la letra de la declaración conciliar Dignitatis Humanae. Se trata de 
una cuestión que, desde distintas perspectivas, ha sido abordada ampliamente por la historiografía. Este texto no pretende, por tanto, hacer ninguna aportación a ese episodio, sino más bien arrojar algo de luz a la trayectoria que, una vez abandonado el apostolado seglar, siguieron algunos de sus miembros.

A pesar de acoger en su seno a buena parte de los antiguos miembros de la Acción Católica (AC), la historia de Promociones de Lecturas Sociedad Anónima (PROLESA) ha pasado casi desapercibida tanto para los estudiosos del postconcilio en España como para los expertos en la Transición. Su origen se sitúa, precisamente, en la oleada de dimisiones de 1968, por lo que cabe conectarlo directamente también con los postulados del Concilio que condujeron a la crisis. Ahora bien, la importancia de esa asociación no radica en su papel durante el cambio político, pues fue prácticamente nulo, sino en su labor de conexión entre dos fenómenos: la crisis de la AC y la Transición española. La existencia de PROLESA permitió a algunos de los miembros del apostolado mantener su compromiso con la sociedad durante los años finales del franquismo, de tal modo que este se mantuvo con vida hasta el comienzo del cambio político. A esto hemos de añadir la amplia red de relaciones con otras asociaciones de carácter político, que permitió a sus miembros iniciar una serie de contactos clave para el nuevo tiempo abierto en noviembre de 1975.

En las siguientes páginas se abordan estos acontecimientos a partir de la figura de Salvador Sánchez-Terán, dirigente juvenil de la Acción Católica a finales de los años cincuenta y presidente de PROLESA entre 1968 y 1973. Ese repaso finalizará con una breve relación de los cargos públicos que ocupó durante la Transición, así como de sus principales aportaciones al proceso de cambio político. Para la elaboración de este trabajo, además de la bibliografía existente sobre la Acción Católica y la recepción del Concilio Vaticano II en España, se ha utilizado el archivo personal del propio SánchezTerán, a lo que hemos de añadir un total de cuatro entrevistas con él que, tras ser grabadas, han sido transcritas. Se incluyen también documentos del Archivo del Ministerio de Fomento, en concreto los legajos de su expediente profesional, y entrevistas a otros protagonistas de la Transición, como es el caso de Rodolfo Martín Villa, Rafael Calvo Ortega y Jaime Lamo de Espinosa. También se incluyen los recuerdos, recogidos durante un encuentro en Boston, del diplomático Pablo SánchezTerán, hermano menor de la figura central de este artículo. 


\section{La Juventud de la Acción Católica}

En febrero de 1959, pocas semanas después de la Conferencia de Metropolitanos de Toledo, la Juventud de la Acción Católica procedió a renovar sus cargos directivos. Como nuevo presidente resultó elegido Salvador Sánchez-Terán, un ingeniero de caminos que se había incorporado al apostolado seglar cuatro años antes ${ }^{1}$. En ese periodo había pasado de ser militante de base a secretario de cursillos y, posteriormente, director ${ }^{2}$. Su ascenso definitivo en la rama juvenil de la AC tuvo como motivo principal el cambio de situación laboral del por entonces presidente, Miguel García Madariaga. Recién finalizados los estudios en Ingeniería de Minas, éste comprendió que su nueva actividad laboral, con un destino lejos de la capital, era incompatible con su cargo. Así fue como se preparó el relevo en la presidencia, que recayó en Sánchez-Terán, quien por entonces había empezado a trabajar como contratado en el Ministerio de la Vivienda ${ }^{3}$.

El nuevo responsable de la Juventud de AC asumía una responsabilidad que, a la postre, iba a resultar clave en el desarrollo de su capacidad de liderazgo. Además, su participación en el Consejo Nacional, máximo órgano del apostolado seglar, le aportó una notable experiencia en reuniones, negociaciones y gestión de órganos directivos ${ }^{4}$. A esto hemos de añadir el contacto con otras personas que, como él, desempeñaban cargos de responsabilidad dentro de la organización:

Mi relación era muy estrecha con los dirigentes masculino, femenino y juvenil femenino, especialmente con Santiago Corral. Con los movimientos especializados también colaboraba, pero la Juventud Obrera Cristiana (JOC) y la Juventud Estudiante Católica (JEC), tenían un gran desarrollo y bastante autonomía. Por el contrario, fuimos nosotros los que impulsamos la Juventud Rural Católica (JARC) y la Juventud Independiente Católica (JIC), que todavía eran débiles a finales de los cincuenta5.

\footnotetext{
${ }^{1}$ Entrevista a Salvador Sánchez-Terán, Madrid, 23 de diciembre de 2010.

2Ibidem: «Primero fui secretario de cursillos, y más adelante director de cursillos. Finalmente, me eligieron presidente nacional de la Juventud de la Acción Católica en febrero de 1959».

3 Entrevista a Salvador Sánchez-Terán, Madrid, 5 de diciembre de 2012.

4 Además de los presidentes masculino y femenino de la Juventud, formaban parte del Consejo Nacional los dirigentes de la rama masculina y femenina de la Acción Católica, los representantes de los movimientos especializados (JOC, JEC, JARC y JIC), y los encargados del Aspirantado, del Secretariado de Cursillos de Cristiandad y de las revistas Signo y Militante.

5 Entrevista a Salvador Sánchez-Terán, Madrid, 5 de diciembre de 2012.
} 
De igual modo, la importancia del organismo que presidía le permitió mantener encuentros con personalidades de la vida política y religiosa del país ${ }^{6}$. En esos años fueron frecuentes las visitas al cardenal primado de Toledo, Enrique Plá y Deniel, que tenían como principal objetivo solicitar respaldo económico para las actividades de la Juventud de AC7. También estableció contacto con el obispo secretario de la Conferencia de Metropolitanos, Vicente Enrique y Tarancón, que por entonces era prelado de Solsona. Ambos iniciaron, a principios de los años sesenta, una relación de estrecha amistad y colaboración que se prolongó durante toda su vida ${ }^{8}$.

Sánchez-Terán permaneció cuatro años en la presidencia juvenil de la AC, un periodo que resultó de suma importancia para su labor política durante la Transición. La necesidad de conocer los diversos grupos locales y de solventar los problemas que pudieran surgir en cada uno, le llevó a realizar numerosos viajes por la Península. En ellos prestaba especial atención a los cuadros dirigentes, básicos en el correcto funcionamiento de un organismo tan numeroso y descentralizado como aquel 9 . Tiempo después, cuando abandonó el apostolado seglar y tomó la decisión de participar en la vida política, muchos de los miembros de aquellos cuadros dirigentes le acompañaron en su nueva empresa:

Cuando se produjo la crisis de la Acción Católica, muchos de esos hombres pasaron a PROLESA. Hablo de Luis Ortiz - que era el secretario general y que más tarde se convirtió en ministro de Obras Públicas-, Alejandro Rebollo - mi subsecretario en Transportes y Comunicaciones-, Vicente Ruiz Monrabal - diputado de la UCD por Valencia -, Armando Benito Calleja - senador por Madrid-, Fernando García Agudín....10.

Precisamente la crisis de la Acción Católica será objeto de análisis en el siguiente epígrafe. Sin embargo, antes de abordarla hemos de hacer referencia, aunque sea brevemente, al nombramiento de Sánchez-Terán como vicepresidente internacional de las Juventudes Católicas. La elección tuvo lugar en una reunión celebrada en Buenos Aires en 1960, cuando apenas llevaba unos meses al frente de la rama juvenil

\footnotetext{
6 Entrevista a Rodolfo Martín Villa, Madrid, 20 de junio de 2012.

7 Entrevista a Pablo Sánchez-Terán, Boston, 21 de junio de 2012: «De vez en cuando, viajaba a Toledo para solicitar al Cardenal Primado Plá y Deniel ayuda económica para los Jóvenes de la Acción Católica. En una ocasión, Salvador me dijo que el Cardenal Plá y Deniel tenía una inteligencia política de primera categoría».

${ }^{8}$ Entrevista a Salvador Sánchez-Terán, Valladolid, 17 de agosto de 2013.

9 Ibidem: «...hizo que recorriera España formando cuadros dirigentes y organizando un grupo tan importante como la Acción Católica. Allí me forjé como dirigente y organizador».

10 Ibidem.
} 
española ${ }^{11}$. Lo más destacable de este nuevo cargo era que llevaba aparejado una serie de responsabilidades que influyeron de manera decisiva en su recepción del Concilio Vaticano II y en su futura labor política. En un estado aislado internacionalmente, Sánchez-Terán viajaba anualmente a París y Roma para asistir a las reuniones del Comité Ejecutivo. A esto hemos de añadir las diversas visitas que realizaba a otras delegaciones de la organización seglar en Europa y América Latina. Estas experiencias, al alcance de muy pocos españoles por aquel entonces, contribuyeron a abrir su mente a nuevas realidades culturales y políticas, al tiempo que le aportaron una visión distinta de las relaciones gobierno-ciudadano.

\section{Sánchez-Terán y la crisis de Acción Católica}

La inserción en un contexto más amplio de la crisis en la que se vio inmerso el apostolado seglar es condición sine qua non para comprender cómo, durante la segunda mitad de los años sesenta, quedó prácticamente desarticulada una organización que contaba con más de medio millón de militantes. En ese escenario hemos de situar, tanto los sucesos de carácter internacional que influyeron de manera profunda en España, como aquellas cuestiones puramente internas en las relaciones Iglesia-Estado. En el primero de ellos destaca el Concilio Vaticano II y la defensa por parte de Roma de la libertad religiosa, la dignidad de la persona humana y la promoción de la justicia social ${ }^{12}$. La convocatoria, hecha pública por Juan XXIII en el consistorio de cardenales del 25 de enero de 1959, fue acogida en el seno de la Acción Católica con una profunda alegría:

La convocatoria del Concilio fue, al menos para mí, una eclosión de alegría. Después todo resultó mucho más complejo, pero creo que esa es la mejor definición de lo que sentimos en la Acción Católica al conocer la noticia de su convocatoria. Desde luego, la defensa de la dignidad humana y de la libertad religiosa fue clave. $\mathrm{Y}$, en parte, eso estuvo detrás de la crisis de la Acción Católica ${ }^{13}$.

Una vez finalizada la fase preparatoria y publicado el motu proprio Superno Dei nutu en 1960, el Concilio fue inaugurado por el pontífice el 11 de octubre de 1962 en la Basílica de San Pedro. Sin embargo, Juan XXIII no llegó a vivir su final. Sería su

\footnotetext{
${ }^{11}$ Entrevista a Salvador Sánchez-Terán, Madrid, 23 de diciembre de 2010.

12 Declaración DignitatisHumanae sobre la libertad religiosa, Concilio Vaticano II.

13 Entrevista a Salvador Sánchez-Terán, Madrid, 5 de diciembre de 2012.
} 
sucesor, Pablo VI, el encargado de clausurarlo tres años después, el 8 de diciembre de 1965. El Vaticano II abría una nueva etapa en la historia de la Iglesia, un periodo no exento de ciertas convulsiones en los primeros años del posconcilio. Estasfueron, si cabe, mayores en el caso español, cuyo régimen político había contado con el apoyo de la jerarquía eclesiástica desde el estallido de la Guerra Civil. De la noche a la mañana, las conclusiones del Concilio dejaban a España fuera de los márgenes políticos establecidos por Roma. Esto obligaba al gobierno a realizar un importante esfuerzo para adecuarse a la nueva realidad. Un trayecto en el que cuestiones como la libertad religiosa, el derecho de presentación o el respeto a los derechos humanos iban a plantear no pocas dificultades entre la Iglesia y el régimen ${ }^{14}$.

La doctrina del Concilio Vaticano II, y especialmente los campos a los que nos acabamos de referir, llevó a una parte significativa de la Iglesia española a distanciarse de los poderes públicos. Ese proceso se había iniciado incluso unos años antes, como bien se aprecia en algunos textos de las revistas de la Acción Católica, Ecclesia y $S$ igno $^{15}$. Es más, en el año 1957, a raíz de un artículo publicado en esta última, el Ministerio de Información inició un proceso contra varios dirigentes juveniles, entre ellos Salvador Sánchez-Terán, por haber expresado ideas aperturistas ${ }^{16}$.

Ahora bien, el proceso de demolición del nacionalcatolicismo no se inició hasta los años del Concilio. El objetivo, que no era otro que despegarse de un régimen al que cada vez más católicos se oponían, se llevó a cabo en dos etapas. En un primer momento el protagonismo correspondió a los laicos y, muy especialmente, a los miembros de la Acción Católica. Después, una vez terminada la crisis del apostolado seglar, fue la propia Jerarquía de la Iglesia española la que comenzó a romper los puentes que la unían al franquismo. Es precisamente en el desfase cronológico entre ambos procesos donde hemos de situar el enfrentamiento entre unos obispos, a los que el régimen presionaba para detener lo que era considerado como una infiltración marxista en la AC, y las asociaciones de laicos:

Pienso que la Jerarquía se sintió presionada por el régimen, que nos consideraba como el germen de un grupo opositor. Los obispos, a los que de hecho no agradaba nuestra autonomía -siempre apostólica, no política-, manifestaron su inquietud

\footnotetext{
14 ANDRÉS-GALLEGO, José, PAZOS, Antón, La Iglesia en la España contemporánea, vol. 2, 1936-1999, Madrid, Encuentro, 1999, pp. 145-146.

${ }^{15}$ SUÁREZ FERNÁNDEZ, Luis, Franco, Barcelona, Ariel, 2005, pp. 506-507.

${ }^{16}$ Entrevista a Salvador Sánchez-Terán, Valladolid, 17 de agosto de 2013.
} 
por nuestra independencia, y sus sospechas de que había algo más que actividad apostólica ${ }^{17}$.

La principal consecuencia de ese enfrentamiento fue la dimisión, en la primavera de 1968, de la inmensa mayoría de los consiliarios y dirigentes nacionales de la Acción Católica. Sin embargo, el proceso se inició mucho antes, en la Asamblea Plenaria de obispos celebrada, el 23 y 24 de julio de 1965, en Santiago de Compostela. En ella, la mayoría de los preladosmostraron su profunda preocupación por la deriva que tomaban los movimientos de seglares, a los que acusaban de actuar al margen de la Jerarquía, así como de participar en actividades ilegales contrarias al régimen ${ }^{18}$. Acusaciones que, si bien exageradas, no carecían de algún fundamento en lo concerniente a ciertos sectoresde la Acción Católica ${ }^{19}$. Ante esta problemática, la asamblea decidió constituiruna Comisión Episcopal para el Apostolado Seglar (CEAS) formada por los obispos de Ciudad Real (Juan Hervás), Sigüenza-Guadalajara (Laureano Castán), Calahorra (Abilio del Campo), y el obispo consiliario de la AC, José Guerra Campos. Para presidir este organismo se designó al arzobispo de Madrid-Alcalá, Casimiro Morcillo.

Tuvieron que pasar casi once meses para que los dirigentes de la ACconocieran, de primera mano, las conclusiones a las que habían llegado los obispos en su reunión de Santiago de Compostela. El escenario del primer desencuentro entre el apostolado seglar y la Jerarquía de la Iglesia española fue el Valle de los Caídos, donde en junio de 1966 se celebraronlas VII Jornadas de la Acción Católica ${ }^{20}$. En un primer momento, la presentación de las comunicaciones se desarrolló en el clima de concordia que había caracterizado a los eventos de años anteriores. Sin embargo, con motivo del discurso de clausura a cargo del presidente de la CEAS, la tranquilidad se fue tornando en crispación, hasta provocar un profundo malestar entre muchos seglares. El arzobispo Casimiro Morcillo aludió en su intervencióna algunas de las conclusiones contenidas en el acta de la Asamblea Plenaria, sobre la que los dirigentes de la Acción Católica no tenían conocimiento ${ }^{21}$.

\footnotetext{
17 Entrevista a Salvador Sánchez-Terán, Madrid, 5 de diciembre de 2012.

18 DE MEER LECHA-MARZO, Fernando, Antonio Garrigues. Embajador ante Pablo VI, Navarra, Thomson-Aranzadi, 2007, p. 69.

19 Entrevista a Salvador Sánchez-Terán, Valladolid, 17 de agosto de 2013.

${ }^{20}$ Las primeras Jornadas de la Acción Católica se celebraron en 1960 y, a partir de esa fecha, la experiencia se repitió con una periodicidad anual. La séptima edición, escenario del desencuentro entre obispos y seglares, llevaba por título «La A. C. a la luz del Concilio».

${ }^{21}$ SÁNCHEZ-TERÁN, Salvador, La crisis de la Acción Católica, in Pablo VI y España, BresciaRoma, Istituto Paolo VI-Studium, 1994, p. 85.
} 
Una semana después de esa escenificaciónpública del desencuentro entre obispos y seglares, la Comisión Permanente de la Conferencia Episcopal volvió a reunirse. Los prelados, en una decisión tomada por unanimidad, rechazaron las conclusiones y el resumen de las Jornadas «por su acusado temporalismo»22 y por contener «afirmaciones y actitudes no compatibles con el espíritu de la AC»23. La realidad que estaba detrás de esa decisión no era otra que la preocupación del episcopado por evitar que los dirigentes del apostolado seglar, aprovechando el cargo que ocupaban hicieran públicas sus ideas en cuestiones de libertad política, sindical y de asociación. En concreto, preocupaba mucho a la Jerarquía la postura de los movimientos especializados, principalmente los jóvenes, en relación a la Ley Orgánica del Estado, que debía someterse a referéndum a finales de $1966^{24}$.

El rechazo de las conclusiones de las Jornadas por parte de la Comisión Permanente del episcopado español, no hizo más que ensanchar el abismo que se abría entre la Jerarquía y los seglares. Sin embargo, el posterior anuncio, por parte de la Conferencia Episcopal, de que se celebraría una Asamblea Plenaria para abordar la cuestión de la Acción Católica, permitió rebajar la tensión existente. De esta manera, se inició un periodo de diálogo entre ambas partes que, no exento de algún sobresalto destaca la dimisión masiva de muchos dirigentes seglares ${ }^{25}$-, sirvió como trabajo preparatorio para la citada reunión. Ésta se celebró, finalmente, entre el 27 de febrero y el 4 de marzo de 1967. Si bien las posturas se mantenían muy alejadas, algunos de los representantes de la AC trataron de tender puentes con los obispos buscando puntos de acuerdo ${ }^{26}$.

En las semanas que siguieron a la Asamblea Plenaria, parecía que la crisis abierta podría cerrarse finalmente gracias al clima de entendimiento que se había generado entre los obispos y los dirigentes del apostolado seglar. Ahora bien, la única mejora sustancial que se había producido con respecto a la polémica desatada tras las Jornadas

${ }^{22}$ ANDRÉS-GALLEGO, José, PAZOS, Antón, op. cit., p. 156.

23 SÁNCHEZ-TERÁN, Salvador, op. cit., p. 85.

24 MONTERO GARCÍA, Feliciano, La Iglesia: de la colaboración a la disidencia (1956-1975), Madrid, Encuentro, 2009, pp. 168-169.

25 El principal enfrentamiento en los meses previos a la Asamblea Plenaria fue la renovación de los consiliarios, prevista por los estatutos, que los militantes interpretaron como una "limpieza", al ser relevados, de manera injustificada y sin consulta a los dirigentes del apostolado seglar, seis de ellos. Como respuesta, algunos cargos juveniles comenzaron a dimitir. A su vez, varios responsables nacionales de las distintas ramas y movimientos enviaron dos cartas - la primera en agosto y la segunda en noviembre - al presidente de la Conferencia Episcopal, el cardenal Quiroga Palacios, mostrando su disconformidad con el curso de los acontecimientos y proponiendo un diálogo que permitiera superar la crisis. Esa voluntad de acuerdo mostrada por los dirigentes permitió frenar la ola de dimisiones y la escalada de tensión, al tiempo que abría las puertas a una posible solución en la futura Asamblea.

${ }^{26}$ SÁNCHEZ-TERÁN, Salvador, op. cit., p. 86. 
del Valle de los Caídos era la voluntad de diálogo entre ambas partes. En el fondo, las profundas discrepancias existentes seguían latentes y se manifestaban en forma de pequeños enfrentamientos que, en cualquier momento, podían agudizar la crisis existente en el seno de la Acción Católica. De hecho, a pesar del relativo apaciguamiento, la Comisión Nacional de la Hermandad Obrera de la Acción Católica (HOAC) mostró, el 23 de abril, su total rechazo al Comunicado Final de la Conferencia Episcopal. Este documento hacía hincapié en la autoridad exclusiva del Magisterio para emitir juicios morales sobre cuestiones políticas, al tiempo que se advertía a los seglares de la Acción Católica que no debían colaborar con los marxistas ${ }^{27}$. No sería el último acto de "rebeldía" del apostolado obrero, pues en marzo del año siguiente la JOC y la HOAC se negaron a aceptar los nuevos Estatutos de la Acción Católica ${ }^{28}$. También los estudiantesmostraron su desacuerdo ante un reglamento que los vinculaba a una Junta Nacional erigida como único órgano directivo de carácter permanente. Hasta tal punto llegó la tensión entre el episcopado y la JEC en relación con los Estatutos, que la rama femenina (JEC/F) dimitió en bloque ${ }^{29}$. El rechazo por parte de los movimientos especializados llevó a la CEAS a excluirlos de los Estatutos de 1968. Tanto la Conferencia Episcopal como su presidente, el obispo Quiroga Palacios, decidieron iniciar con ellos un diálogo por separado con el fin de encontrar otra vía más adecuada a esa realidad ${ }^{30}$. Ésta se alcanzó en febrero de 1970, con su reconocimiento como movimientos especializados de la AC desvinculados de los órganos centrales del apostolado seglar.

$\mathrm{Al}$ margen del conflicto con el apostolado obrero y estudiantil, es necesario indicar que, a pesar de aceptar los nuevos Estatutos, la Acción Católica General consideró poco satisfactorio el resultado de los trabajos. Así se lo comunicaron sus dirigentes a los obispos, con los que habían mantenido un intenso diálogo durante todo el proceso de redacción. No en vano, en las reuniones mantenidas en los días previos con la CEAS ya se habían hecho evidentes las discrepancias en materia de elección y nombramiento de los dirigentes, dirección, declaraciones sobre cuestiones sociales, y comunicación. Por ese motivo, aprovechando la celebración en Roma del Congreso Mundial de Apostolado Seglar los dirigentes de la Acción Católica decidieron llevar la problemática ante la Santa Sede. En octubre de 1967, una comisión presidida por Santiago Corral se reunió en dos ocasiones con mons. Giovanni Benelli. El sustituto de la Secretaría de Estado del

27 MONTERO GARCÍA, Feliciano, op. cit., p. 169.

${ }_{28}$ CALlAHAN, William J., La Iglesia Católica en España (1875-2002), Barcelona, Crítica, 2003, p. 404.

29 SÁNCHEZ-TERÁN, Salvador, op. cit., p. 87.

30 ANDRÉS-GALLEGO, José, PAZOS, Antón, op. cit., p. 156. 
Vaticano escuchó con atención las reclamaciones de los seglares, al tiempo que se comprometía a informar de ello a Pablo VI. La mediación de la Santa Sede hizo posible que la Conferencia Episcopal Española reformara aquellos artículos de los Estatutos que, desde la Acción Católica, se consideraban inaceptables. Finalmente, en la VI Asamblea Plenaria del Episcopado, celebrada el 28 de noviembre de 1967, fueron aprobados los nuevos Estatutos, que debían entrar en vigor el 1 de febrero del año siguiente.

A pesar de los acuerdos alcanzados, la crisis de la Acción Católica siguió su curso. No en vano, como se ha indicado más arriba, los movimientos especializados rechazaron los nuevos Estatutos, mientras que las distintas ramas de la modalidad general los consideraron poco satisfactorios. De esta manera, el éxodo de militantes que se había iniciado a raíz de las Jornadas de 1966, lejos de detenerse, se incrementó. A esto hemos de añadir el malestar y hastío de los dirigentes, que llevaban casi dos años luchando por mantener con vida una estructura que, por la manifiesta falta de entendimiento con la CEAS, hacía aguas. Su frustración llegó al máximo cuando, en sus conversaciones con los obispos, fueron conscientes de que la nueva Acción Católica que se pretendía construir era muy distinta a aquella en la que ellos habían colaborado desde la década de los cincuenta. A pesar de todo, antes de presentar la dimisión, decidieron agotar todas las vías de diálogo. En marzo y abril de 1968, una comisión formada por Santiago Corral, Carmen Victory, Antonio García Pablos, Francisca Majó, Enrique del Río y Salvador Sánchez-Terán se reunió en varias ocasiones con el presidente de la CEAS. Sin embargo, la distancia entre ambas partes era tan grande que el entendimiento no fue posible:

En el fondo lo que subyacía era una visión drásticamente distinta de la Acción Católica, los jóvenes apostaban por una Iglesia comprometida con el Vaticano II, la justicia social y la democracia, mientras que una parte poderosa de la Jerarquía Eclesial seguía anclada en posturas preconciliares ${ }^{31}$.

En mayo se inició una oleada de renuncias que incluyó a más de cien dirigentes nacionales, cuyo ejemplo fue seguido por numerosos cargos diocesanos y locales ${ }^{32}$. A su vez, Santiago Corral redactó una carta dirigida al cardenal Quiroga Palacios en la que exponía las causas de su dimisión. Esta iniciativa fue imitada por los presidentes de las juntas nacionales, así como de las distintas ramas y movimientos de la Acción Católica:

${ }^{31}$ Entrevista a Salvador Sánchez-Terán, Valladolid, 17 de agosto de 2013. $3^{2}$ ANDRÉS-GALLEGO, José, PAZOS, Antón, op. cit., pp. 156-157. 
Presentamos nuestra dimisión con Santiago Corral a la cabeza. Para no hacer mucho ruido y no aumentar el daño a la Iglesia y al apostolado seglar, se decidió no hacer una dimisión colectiva con un documento público explicativo. Los presidentes de Juntas, Ramas o Movimientos enviamos una breve carta individual al Presidente de la Conferencia Episcopal 33.

\section{El nacimiento de PROLESA y su papel en la Transición}

La crisis de la Acción Católica llevó a buena parte de sus miembros a dedicar a otras tareas los esfuerzos que, hasta el momento, dedicaban al apostolado seglar. Tras la oleada de renuncias de 1968, algunos orientaron su actividad hacia la política, colaborando en iniciativas existentes o formando sus propios grupos. Ese fue el caso de Salvador Sánchez-Terán que, junto a sus principales colaboradores de la rama juvenil, fundó Promociones de Lecturas Sociedad Anónima, más conocida como PROLESA. Con sede en el número 11 de la calle Fernando VI, esta asociación servía como tapadera para la constitución de un grupo político alternativo al régimen franquista y de apertura hacia la democracia34. Si bien, de manera oficial no era más que un conjunto de personas dedicadas a la crítica de libros, la finalidad real de PROLESA era participar de manera activa en la vida política del país. De hecho, Sánchez-Terán y sus colaboradores se esforzaron por entrar en contacto con otros grupos similares, así como con personalidades públicas afines a su causa 35 .

La fundación de PROLESA se llevó a cabo a las pocas semanas de producirse la dimisión de Santiago Corral y de sus principales colaboradores en la Acción Católica. En esa primera reunión quedó constituida la asociación, de la que Salvador SánchezTerán pasó a ser presidente. Junto a él, por su importancia a lo largo de la Transición, cabe destacar a los siguientes miembros: Luis Ortiz, Alejandro Rebollo, Vicente Ruiz Monrabal, Armando Benito Calleja, Demetrio Madrid, Fernando García Agudín y David León ${ }^{36}$. A partir de ese primer encuentro, los fundadores de PROLESA comenzaron a

33 SÁNCHEZ-TERÁN, Salvador, op. cit., p. 89.

34 Entrevista a Salvador Sánchez-Terán, Madrid, 23 de diciembre de 2012: «Recuerdo una escena un tanto cómica que sucedió cuando fuimos a inscribir PROLESA en el registro de asociaciones. El notario leyó los papeles, nos miró un buen rato con gesto sonriente, y soltó una frase irónica para hacernos entender que sabía de sobra que aquello era algo más que una sociedad para la promoción de la lectura».

35 Ibidem.

${ }^{36}$ Los socios de PROLESA, además de los ya mencionados, fueron: Manuel Álvarez Fuentes, José Luis Álvarez López, Félix Álvarez-Miranda, José Ignacio Añas Bote, Pedro Aramburu, 
recorrer las distintascapitales de provincia en busca de nuevos socios. Una vez allí, reunían a los antiguos miembros de la Acción Católica y les proponían integrarse en ese nuevo proyecto. En definitiva, la relación entre la crisis del apostolado seglar y la aparición de Promociones de Lecturas S. A. no se circunscribe únicamente al marco cronológico, sino también a los contactos que sirvieron para formar el primer grupo de socios. Ahora bien, eso no nos debe llevar a concluir que se trataba de un ente nacido en el seno de la Acción Católica: PROLESA, a pesar de la íntima relación que guardaba con el apostolado seglar, fue posterior a su crisis.

\begin{abstract}
He de indicar que nunca nos consideramos el germen de un futuro movimiento político, democristiano al estilo germano-italiano o del tipo que fuera. La demostración de esto es que, al hundirse la Acción Católica, cada uno siguió caminos distintos, tanto en la política como fuera de ella. Unos se integraron en movimientos de derecha, otros de centro y otros de izquierda; y muchos no se dedicaron a la política. Más bien diría que fue la crisis de la Acción Católica la que nos empujó a la política37.
\end{abstract}

A pesar de poseer, por la presencia de algunos representantes del apostolado obrero, una importante carga de preocupación social, PROLESA era, por sus orígenes, un grupo de marcado carácter democristiano. Ahora bien, la presencia de antiguos militantes de la JOC y la HOAC le aportaba cierta pluralidad de sensibilidades políticas que resultó determinante en la existencia de contactos con grupos de izquierdas, además de los que ya mantenían con otros de centro-derecha. En sus cinco años de existencia, el único partido opositor con el que PROLESA no mantuvo contacto fue el comunista. Liberales, democristianos, socialdemócratas, socialistas y nacionalistas tuvieron encuentros con Sánchez-Terán y los restantes componentes de la directiva.

Vicente Ausín, José Antonio Azuares Candas, Santiago Baños Vales, Armando Calleja Benito, José Luis Carreras, Telésforo Carrión, Gonzalo Casado Herce, Mariano de Castro Morcillo, Ángeles Corrales Gutiérrez, María José Díaz Fierros, José de Jaime Eslava, Marta Fernández Torres, Luis Manuel García Mayordomo, Ginés Garrido, José Carlos Gómez Borrero, Santiago González Álvarez-Buylla, Blas González Onrubia, Adriana Gutiérrez Vázquez, Santiago Gutiérrez Vázquez, Sara Guzmán Herrero, Ángel Hernández, Rafael Hevia Rodríguez, José Inglés Falceto, Laureano Lázaro Araujo, David León Blanco, Cristino Lobillo, Antonio Lorente del Prisco, Gonzalo de Lucas Ruiz, José de Lucas Ruiz, Luis Madrigal, Lucas Martín Pantoja, Francisco Martínez, Ángel Ramón Martínez Marín, Juan José Martínez Quesada, José Mercader Canabá, Miguel Muñoz Gea, Fernando Oliván García, Francisco Panadero, José Pérez, Vicente Pinel, Roque Pozo Tamames, Ricardo Reinosa, Fernando de la Rica, María Riestra Pérez, Vicente Ruiz Monrabal, Ricardo Sáinz, Pascual Sánchez Soria, Miguel Ángel Sánchez-Terán, Félix Soria Conde, Miguel Ángel Torremocha, Juan Torres Piñón, Mario Valdivieso Mateo, Ramón Valdivieso Mateo, José Vergara y Goya Villanueva.

37 Entrevista a Salvador Sánchez-Terán, Madrid, 5 de diciembre de 2012. 
Simplificando mucho, podríamos decir que la ideología de PROLESA estaba cercana a la democracia cristiana, pero una democracia cristiana de corte muy abierto, donde cabían postulados socialdemócratas. Eso explica la presencia de personajes como Demetrio Madrid, que había presidido la JOC en Zamora, o nuestros contactos con algunas personalidades del PSOE38.

A esto hemos de añadir el contacto con algunas de las principales personalidades políticas del aperturismo político e, incluso, de la oposición. Nos referimos fundamentalmente a Joaquín Ruiz Giménez, Dionisio Ridruejo, Gregorio Peces-Barba, Alejandro Rojas Marcos, José María de Areilza o Manuel Fraga Iribarne39. Precisamente, para el desarrollo de la sociedad y para la actividad política de varios de sus miembros durante la Transición, fue de una importancia capital la amistad que surgió entre este último y Sánchez-Terán. En sus reuniones con los miembros de PROLESA, Manuel Fraga descubrió un importante grupo de jóvenes interesados en la política que estabandirigidos por una persona con notables capacidades para la organización ${ }^{40}$. Por su parte, Sánchez-Terán se percató pronto de que Fraga era la única persona de su entorno que, en aquel momento, tenía un programa conocido para llevar a cabo el cambio político ${ }^{41}$. Todo eso llevó a que, tanto durante los últimos años de la vida de Franco como a lo largo de la Transición, ambos se esforzaran por fortalecer sus vínculos políticos:

Podríamos decir que tenía muchas esperanzas en su proyecto, ya que suponía cambiar muchas cosas de las Leyes Fundamentales para llegar a una democracia

${ }^{38}$ Entrevista a Salvador Sánchez-Terán, Madrid, 23 de diciembre de 2010.

39 Entrevista a Salvador Sánchez-Terán, Madrid, 23 de marzo de 2012: «PROLESA fue una asociación parapolítica, bajo la tapadera de sociedad anónima que vendía libros, creada por exdirigentes de los movimientos juveniles de Acción Católica. Estuvo operativa de 1968 a 1973 bajo mi presidencia. El objetivo era establecer el contacto con organizaciones y dirigentes que promovieran un sistema democrático para nuestro país. Ello nos llevó a tener reuniones con grupos socialdemócratas, socialistas, liberales, democristianos, regionalistas o nacionalistas vascos y catalanes y con personas como Ruiz Giménez, Dionisio Ridruejo, Peces-Barba, Manuel Fraga, Rojas Marcos o Areilza».

40 Entrevista a Jaime Lamo de Espinosa, Madrid, 20 de julio de 2012: «...era muy todoterreno por su capacidad de gestión y de organización. Y eso, muchas veces, los políticos no lo tienen. Hay muchas veces que el político es ideólogo, creativo, se le ocurren cosas... pero la capacidad de gestionar el día a día, de organizar, de llevar el método y la sistemática de la que hablábamos antes, Salvador la ha tenido siempre».

${ }^{41}$ Entrevista a Pablo Sánchez-Terán, Madrid, 21 de junio de 2012: «El encuentro de Manuel Fraga y Salvador fue un caso de mutuo enamoramiento político, de amor a primera vista político. Desde entonces, Salvador siempre ha tenido un considerable afecto, respeto y admiración por Manuel Fraga». 
equiparable a las del resto de Europa. Y, al mismo tiempo, él creía en mí y en el grupo de jóvenes que yo representaba: los más de cien hombres de PROLESA42.

PROLESA mantuvo una intensa actividad entre 1968 y 1973, periodo en el que su red de contactos no dejó de aumentar. Sin embargo, al asumir Sánchez-Terán la Subsecretaría de Obras Públicas en $1973^{43}$, el grupo se quedó, de hecho, sin dirección efectiva44. De esta manera, se fue disolviendo en los últimos meses del franquismo, hasta que, al constituirse en 1976 y 1977 las distintas agrupaciones políticas, la mayor parte de los socios se integraron en algún partido - principalmente la UCD y el PSOE- $u$ optaron por retirarse de la actividad pública. Así fue como, al iniciarse la Transición, dejó de existir una organización que, desde 1973, apenas registraba actividad.

Ahora bien, la importancia de PROLESA no radica tanto en la labor realizada por el grupo a lo largo de esos años como en su papel de mediación, de puente, entre los "náufragos" de la Acción Católica y el proceso de cambio político.Sirva de ejemplo el caso de Salvador Sánchez-Terán, cuyo desempeño en la Transición es ampliamente reconocido por la historiografía. El que fuera presidente de PROLESA estuvo presente, a partir de 1976, en algunos de los acontecimientos claves de la democratización. No en vano, fue gobernador civil de Barcelona entre febrero de 1976 y abril de 1977 y, en calidad de representante del Gobierno de España, negoció con el honorable Josep Tarradellas el restablecimiento de la Generalitat de Cataluña45. Además, durante la primera legislatura, fue miembro de la Comisión Constitucional del Congreso, secretario de Organización de la UCD y uno de los firmantes de los Pactos de la Moncloa. A todo esto hemos de añadir su presencia en tres de los gobiernos de Adolfo Suárez, así como su labor al frente de la Compañía Telefónica entre 1980 y 1982. En resumen, participó activamente en el proceso de modernización y cambio político experimentado por el país entre mediados de los años setenta y principios de los ochenta. En esa tarea le acompañaron algunos ex miembros de PROLESA, de entre los que cabe destacar a Alejandro Rebollo, subsecretario de Transportes y Comunicaciones entre 1978 y 1980, así como miembro destacado de la UCD.

${ }^{42}$ Entrevista a Salvador Sánchez-Terán, Madrid, 5 de julio de 2012.

43 Decreto 1459/1973, de 5 de julio de 1973 (BOE, 6 julio 1973), por el que se nombra Subsecretario de Obras Públicas a don Salvador Sánchez-Terán Hernández, Archivo del Ministerio de Fomento, Expediente de Salvador Sánchez-Terán Hernández, legajo 38.829, p. 27.

44 Entrevista a Salvador Sánchez-Terán, Madrid, 5 de diciembre de 2012: «Las responsabilidades como director general de RENFE y, más tarde, como subsecretario de Obras Públicas, me fueron alejando poco a poco de PROLESA. De hecho, a todos nos pasó algo similar, hasta que desapareció».

45 Entrevista a Rafael Calvo Ortega, Madrid, 15 de abril de 2013: «La "Operación Tarradellas" es lo más brillante que yo recuerdo de él». 


\section{Conclusiones}

A lo largo de las páginas anteriores se han expuesto algunas de las principales consecuencias que, para el régimen español, trajo consigo la declaración Dignitatis Humanae. Las dificultades del oficialmente católico franquismo para adaptarse a las novedades del Concilio, llevó a que se produjeran varios enfrentamientos con la Santa Sede. De igual manera, el episcopado español, formado mayoritariamente en la doctrina del nacionalcatolicismo, tuvo que hacer frente a un apostolado seglar que abogaba por la separación entre Iglesia y Estado. Tanto esas cuestiones, como sus repercusiones en el proceso de cambio político iniciado en 1975, han sido abordadas desde distintas perspectivas en la bibliografía existente.Ahora bien, quizá por la escasez de fuentes, la historiografía ha ignorado el papel de PROLESA; una asociación surgida a raíz de la crisis de la Acción Católica que, en los años finales del régimen, mantuvo contactos con los principales grupos y dirigentes políticos aperturistas y reformistas. Es más, aunque el papel de algunos de sus socios durante la Transición es bien conocido, no se menciona su pertenencia a PROLESA.

El caso paradigmático es el de Salvador Sánchez-Terán, cuya trayectoria en el apostolado seglar ha servido como guía para el desarrollo de este artículo. Al analizarlo nos encontramos con un importante dirigente juvenil que, tras abandonar la Acción Católica, pasó a presidir una asociación de carácter político, dedicando a esa tarea los esfuerzos que previamente destinaba al apostolado. A su vez, en el origen del proceso que le condujo a dimitir de sus cargos hemos de situar, como uno de los principales motivos, la recepción del Concilio Vaticano II. Las dificultades para ponerlo en práctica en la AC condujo, tanto Sánchez-Terán como a otros miembros del apostolado seglar, a buscar otro ámbito donde hacerlo. Este resultó ser, a la postre, la política, plasmada primero en una asociación que mantenía contactos con grupos y personas afines y, más tarde, en una no desdeñable labor de colaboración en la construcción del edificio de la Transición.

En definitiva, a la relación causa-efecto que se ha señalado entre la crisis de la Acción Católica y el origen de PROLESA, habría que añadir la importancia de esta última en el nacimiento de la vocación política de varios de sus miembros y, en concreto, de Sánchez-Terán.En lo que a este último respecta, hay que señalar la importancia decisiva que tuvieron, en su labor pública, los planteamientos expuestos en la Dignitatis Humanae. De esta manera, si bien es evidente la existencia de una 
ruptura entre la tarea apostólica previa y la posterior actividad política, se aprecia una continuidad en cuestiones como la libertad religiosa, la dignidad de la persona humana y la promoción de la justicia social. Principios que, en buena medida, quedaron ampliamente recogidos en la Constitución de 1978. 


\section{* El autor}

Carlos González Martínez es licenciado (2007) y doctor en Historia (2014) por la Universidad de Valladolid con la tesis Salvador Sánchez-Terán, una biografía política (1934-1982), dirigida por el catedrático Pablo Pérez López. También es licenciado en Ciencias Políticas (2014). En la actualidad está finalizando sus estudios de grado en Sociología y estudia la labor política de Rafael Calvo Ortega dentro del Grupo de Investigación en Historia Reciente (GIHRE). Entre sus publicaciones destacan: «La intervención estatal en el Metro de Madrid (1980-1982)», in Historia Actual : Modernización Urbana en la España Contemporánea, 2010; Salvador Sánchez-Terán, presidente de Telefónica (1980-1982), in ORTEGA LÓPEZ, Teresa María, DEL ARCO BLANCO Miguel Ángel (eds.), Claves del mundo contemporáneo : debate e investigación: actas del XI Congreso de la Asociación de Historia Contemporánea, Granada, Comares, 2013; colaborador en Diccionario biográfico de parlamentarios españoles. 18201854, Madrid, Cortes Generales, 2013; colaborador en Elites parlamentarias de Castilla la Vieja y León en las Cortes Generales (1810-1874), Valladolid, Universidad de Valladolid, 2014.

URL: < http://www.studistorici.com/progett/autori/\#GonzalezMartinez >

\section{Per citare questo articolo:}

GONZÁLEZ MARTíNEZ, Carlos, «De la Dignitatis Humanae a la democracia: la crisis de la Acción Católica y el nacimiento de PROLESA (1965-1978)», Diacronie. Studi di Storia Contemporanea : Un bilancio della scommessa democratica della Chiesa cattolica, 29/06/2016,

URL:<http://www.studistorici.com/2016/06/29/gonzalez-martinez_numero_26/ >

Diacronie Studi di Storia Contemporanea $\vartheta$ www.diacronie.it

Risorsa digitale indipendente a carattere storiografico. Uscita trimestrale. redazione.diacronie@hotmail.it

Comitato di redazione: Jacopo Bassi - Luca Bufarale - Elisa Grandi - Antonio César Moreno Cantano - Deborah Paci - Fausto Pietrancosta - Alessandro Salvador - Matteo Tomasoni - Luca Zuccolo

Diritti: gli articoli di Diacronie. Studi di Storia Contemporanea sono pubblicati sotto licenza Creative Commons 3.0. Possono essere riprodotti e modificati a patto di indicare eventuali modifiche dei contenuti, di riconoscere la paternità dell'opera e di condividerla allo stesso modo. La citazione di estratti è comunque sempre autorizzata, nei limiti previsti dalla legge. 